University of Nebraska - Lincoln

DigitalCommons@University of Nebraska - Lincoln

November 1997

\title{
Dispersion coefficients for highly excited molecular states of $\mathrm{K}_{2}$
}

Mircea Marinescu

University of Nebraska - Lincoln

Anthony F. Starace

University of Nebraska-Lincoln, astarace1@unl.edu

Follow this and additional works at: https://digitalcommons.unl.edu/physicsstarace

Part of the Physics Commons

Marinescu, Mircea and Starace, Anthony F., "Dispersion coefficients for highly excited molecular states of $\mathrm{K}_{2}$ " (1997). Anthony F. Starace Publications. 62.

https://digitalcommons.unl.edu/physicsstarace/62

This Article is brought to you for free and open access by the Research Papers in Physics and Astronomy at DigitalCommons@University of Nebraska - Lincoln. It has been accepted for inclusion in Anthony F. Starace Publications by an authorized administrator of DigitalCommons@University of Nebraska - Lincoln. 


\title{
Dispersion coefficients for highly excited molecular states of $\mathbf{K}_{2}$
}

\author{
Mircea Marinescu and Anthony F. Starace \\ Department of Physics and Astronomy, The University of Nebraska, Lincoln, Nebraska 68588-0111
}

(Received 29 May 1997)

\begin{abstract}
We report results of theoretical calculations for the dispersion coefficients associated with the long-range interaction of two ${ }^{39} \mathrm{~K}$ atoms in the states ${ }^{39} \mathrm{~K}(4 s)-{ }^{39} \mathrm{~K}(5 d, 6 d, 7 s)$. These results have been found by Wang et al. [Phys. Rev. Lett. 78, 4173 (1997)] to be in a good agreement with their measurements.

[S1050-2947(97)10010-5]
\end{abstract}

PACS number(s): $34.20 . \mathrm{Cf}, 31.15 . \mathrm{Md}$

\section{INTRODUCTION}

Progress in ultracold spectroscopy has made possible experimental investigation of weakly bound highly excited molecular states with an unprecedent level of accuracy. In particular, the first observation of optical-optical doubleresonance photoassociative spectroscopy of ultracold ${ }^{39} \mathrm{~K}$ atoms near the highly excited ${ }^{39} \mathrm{~K}(4 s)$ $+{ }^{39} \mathrm{~K}(4 d, 5 d, 6 d, 6 s, 7 s)$ asymptotes in a high-density magneto-optical trap has recently been reported [1]. Based on the measurements of the higher excited vibrational levels, it was possible to estimate the values of the dominant dispersion coefficients for the long-range interaction of two $\mathrm{K}$ atoms with one of them in a highly excited state [1]. Theoretical calculations of the dispersion coefficients for the ${ }^{39} \mathrm{~K}(4 s)-{ }^{39} \mathrm{~K}(6 s, 4 d)$ dissociation limits of $\mathrm{K}_{2}$ have been reported previously [2]. There is good agreement with new experimental data for these states [1]. However, no theoretical predictions exist for the higher excited states measured recently [1]. In this Brief Report we present theoretical calculations of the dispersion coefficients for the ${ }^{39} \mathrm{~K}(4 s)-{ }^{39} \mathrm{~K}(7 s, 5 d, 6 d)$ dissociation limits and compare with the experimental measurements [1]. Throughout this paper we use atomic units $\left(\hbar=m_{e}=e=1\right)$.

\section{MATHEMATICAL BACKGROUND}

Derivations of the formal expressions for the dispersion coefficients associated with the $S-S$ and $S-D$ dissociation limits were presented in Ref. [2], based on a model potential description of the active valence electron of the alkali-metal atoms [3]. Here we therefore only present the final results needed for our analysis. Briefly, in the long-range limit, the molecular potential interaction may be written as a series of inverse powers of the internuclear distance $R$. The coefficients of this series are the dispersion coefficients. They may be computed using perturbation theory, where the perturbation is given by the Coulomb interaction between the atomic charge distributions. For the $S$-S asymptote, where in the dissociation limit one of the atoms is in the ground state $\left\{n_{g}, 0,0\right\}$ and the other in an excited $S$ state $\left\{n_{e}, 0,0\right\}$, the long-range potential interaction is given by the following expression:

$$
V^{\beta}(R)=-\frac{C_{6}^{\beta}}{R^{6}}-\frac{C_{8}^{\beta}}{R^{8}}-\frac{C_{10}^{\beta}}{R^{10}}-\cdots,
$$

where the coefficients $C_{n}^{\beta}$ are the dispersion coefficients, which are obtained from second-order perturbative corrections to the molecular energies. In Eq. (2.1) we have $\beta=p \sigma$, where $p= \pm 1$ for even $(g)$ and odd $(u)$ molecular states, and $\sigma= \pm 1$ for singlet and triplet states. ${ }^{1}$ The dispersion coefficients $C_{6}^{\beta}, C_{8}^{\beta}$, and $C_{10}^{\beta}$ are given by the following expressions [2]:

$$
\begin{gathered}
C_{6}^{\beta}=\frac{2}{3}\left(S_{1}^{0}+\beta S_{2}^{0}\right), \\
C_{8}^{\beta}=S_{3}^{0}+S_{4}^{0}+2 \beta S_{5}^{0}, \\
C_{10}^{\beta}=\frac{14}{5} S_{6}^{0}+\frac{4}{3}\left(S_{7}^{0}+S_{8}^{0}\right)+\beta\left(\frac{14}{5} S_{9}^{0}+\frac{8}{3} S_{10}^{0}\right),
\end{gathered}
$$

where $S_{j}^{0}$ for $j=1, \ldots, 10$ are sums of different products of atomic radial matrix elements divided by appropriate energy denominators. $S_{j}^{0}$ are given by Eqs. (46), (47), (50)-(52) and (55)-(59) of Ref. [2]. ${ }^{2}$ Similarly, for the $S$ - $D$ asymptote, where in the dissociation limit one atom is in the ground state and the other is in an excited $D$ state $\left\{n_{e}, 2, m_{e}\right\}$, the long-range interaction potential is given by

$$
V^{m_{e} \beta}(R)=-\frac{C_{5}^{m_{e} \beta}}{R^{5}}-\frac{C_{6}^{m_{e} \beta}}{R^{6}}-\cdots,
$$

where the $C_{5}$ coefficients are obtained in the first order of perturbation and the $C_{6}$ coefficients are obtained in the second order of perturbation. They are given by the following expressions:

$$
C_{5}^{m_{e} \beta}=\frac{(-1)^{m_{e}+1} \beta}{5}\left(\begin{array}{c}
4 \\
2+m_{e}
\end{array}\right)\left(n_{e} 2\left|r^{2}\right| n_{g} 0\right)^{2}
$$

\footnotetext{
${ }^{1}$ In general, for dissociation limits where one atom is in the ground state and the other is in an excited state $\left\{n_{e}, l_{e}, m_{e}\right\}$, we have $\beta=(-1)^{l_{e}} p \sigma$.

${ }^{2}$ Note that there is a misprint in Eq. (56) of Ref. [2]: $E_{n_{3} 0}$ in the denominator should be $E_{n_{e} 0}$.
} 


$$
C_{6}^{m_{e} \beta}=A_{1}^{m_{e}} S_{1}^{2}+A_{2}^{m_{e}} S_{2}^{2}+\beta A_{3}^{m_{e}} S_{3}^{2},
$$

where the algebraic coefficients $A_{j}^{m_{e}}$ for $j=1,2,3$ are given in Table II of Ref. [2], the sums $S_{j}^{2^{j}}$ for $j=1,2,3$ are given by Eqs. (78)-(80) of Ref. [2], and the $\left(\begin{array}{l}n \\ k\end{array}\right)$ are the binomial coefficients. The main task in computing the dispersion coefficients consists in evaluating each of the double sums $S_{1}^{0}, \ldots, S_{10}^{0}$ and $S_{1}^{2}, S_{2}^{2}, S_{3}^{2}$. They are sums over the principal quantum numbers of both of the atoms. For example, the sum $S_{1}^{0}$ is given by

$$
S_{1}^{0}=\sum_{n m} \frac{\left(n 1|r| n_{g} 0\right)^{2}\left(m 1|r| n_{e} 0\right)^{2}}{E_{n 1}+E_{m 1}-E_{n_{e} 0}-E_{n_{g} 0}} .
$$

In general, an efficient method of computation is to separate these sums into independent contributions of each atom by using the following integral representation [4] for each of the denominators in the expressions for the $S$ sums:

$$
\frac{1}{a+b}=\frac{2}{\pi} \int_{0}^{\infty} d \omega \operatorname{Re}\left[\frac{1}{a-i \omega}\right] \operatorname{Re}\left[\frac{1}{b-i \omega}\right]
$$

This integral representation is valid only if $a>0$ and $b>0$. By inverting the sums over the principal quantum numbers and the integral over frequency, and formally computing the sums, we obtain an integral over a product of atomic radial matrix elements containing a radial Green's function for complex energy. The main advantage of this procedure is that the two-center molecular problem is reduced to the onecenter atomic problem.

An example will serve to illustrate the theoretical procedure just described. Consider again the $S_{1}^{0}$ sum given by Eq. (2.8). For the specific order of the energy levels of K, i.e., $4 s, 4 p, 5 s, 3 d, 5 p, 4 d, 6 s, 4 f, 6 p, 5 d, 7 s, 5 f, 5 g, 7 p, 6 d$, etc., it is not always possible to arrange the denominators in the expression of the $S$ sums such that $a>0$ and $b>0$, as required in order to use Eq. (2.9). The cases where the condition $a>0$ and $b>0$ cannot be satisfied must be treated separately. Thus for the energies in $S_{1}^{0}$, if we chose $a=E_{n 1}-E_{4 S}$ and $b=E_{m 1}-E_{7 S}$, then for $\{m 1\}$ $\equiv 4 P, 5 P$, and $6 P$ we have $b<0$. In those cases where $b$ is smaller than 0 , we replace $1 /(a+b)$ by

$$
\frac{1}{a+b}+\frac{1}{a-b}+\frac{2}{\pi} \int_{0}^{\infty} d \omega \operatorname{Re}\left[\frac{1}{a-i \omega}\right] \operatorname{Re}\left[\frac{1}{b-i \omega}\right]
$$

where the last two terms in Eq. (2.10) cancel each other. ${ }^{3}$ This procedure enables us to obtain a complete sum over the atomic states in the integral. The first two terms resulting from Eq. (2.10) are treated separately. Thus, the final expression for $S_{1}^{0}$ is given by

\footnotetext{
${ }^{3}$ This cancellation follows for $b<0$ from the fact that $(a-b)^{-1}=(a+|b|)^{-1}$ and the fact that $\operatorname{Re}(b-i \omega)^{-1}$ $=-\operatorname{Re}(|b|-i \omega)^{-1}$.
}

TABLE I. Dispersion coefficients in a.u. for the ${ }^{39} \mathrm{~K}(4 s)-{ }^{39} \mathrm{~K}(7 s)$ asymptote.

\begin{tabular}{lccc}
\hline \hline$\beta$ & $C_{6} \times 10^{-5}$ & $C_{8} \times 10^{-9}$ & $C_{10} \times 10^{-12}$ \\
\hline+1 & 3.9195 & 1.0217 & 2.8799 \\
-1 & $3.7 \pm 0.2^{\mathrm{a}}$ & & \\
& 3.9226 & 1.0219 & 2.8800 \\
\hline
\end{tabular}

${ }^{\mathrm{a}}$ Measurement of Wang et al. [1].

$$
\begin{aligned}
S_{1}^{0}= & \frac{2}{\pi} \int_{0}^{\infty} d \omega \operatorname{Re}\left[\left(4 S\left|r g_{P}\left(E_{4 S}+i \omega\right) r\right| 4 S\right)\right] \\
& \times \operatorname{Re}\left[\left(7 S\left|r g_{P}\left(E_{7 S}+i \omega\right) r\right| 7 S\right)\right] \\
& +(4 P|r| 7 S)^{2}\left[\left(4 S\left|r g_{P}\left(E_{4 S}-E_{4 P}+E_{7 S}\right) r\right| 4 S\right)\right. \\
& \left.+\left(4 S\left|r g_{P}\left(E_{4 S}+E_{4 P}-E_{7 S}\right) r\right| 4 S\right)\right] \\
& +(5 P|r| 7 S)^{2}\left[\left(4 S\left|r g_{P}\left(E_{4 S}-E_{5 P}+E_{7 S}\right) r\right| 4 S\right)\right. \\
& \left.+\left(4 S\left|r g_{P}\left(E_{4 S}+E_{5 P}-E_{7 S}\right) r\right| 4 S\right)\right] \\
& +(6 P|r| 7 S)^{2}\left[\left(4 S\left|r g_{P}\left(E_{4 S}-E_{6 P}+E_{7 S}\right) r\right| 4 S\right)\right. \\
& \left.+\left(4 S\left|r g_{P}\left(E_{4 S}+E_{6 P}-E_{7 S}\right) r\right| 4 S\right)\right],
\end{aligned}
$$

where $g_{P}$ is the radial Green's function for angular momentum $l=1$. The last three terms in Eq. (2.11) stem from the first two terms in Eq. (2.10) for the three cases where $b<0$. Alternatively, if we choose $a=E_{m 1}-E_{4 S}$ and $b=E_{n 1}-E_{7 S}$, then we obtain

$$
\begin{aligned}
S_{1}^{0}= & \frac{2}{\pi} \int_{0}^{\infty} d \omega \operatorname{Re}\left[\left(4 S\left|r g_{P}\left(E_{7 S}+i \omega\right) r\right| 4 S\right)\right] \operatorname{Re}\left[\left(7 S \mid r g_{P}\left(E_{4 S}\right.\right.\right. \\
& +i \omega) r \mid 7 S)]+(4 P|r| 4 S)^{2}\left[\left(7 S \mid r g_{P}\left(E_{4 S}-E_{4 P}\right.\right.\right. \\
& \left.\left.\left.+E_{7 S}\right) r \mid 7 S\right)+\left(7 S\left|r g_{P}\left(E_{4 S}+E_{4 P}-E_{7 S}\right) r\right| 7 S\right)\right] \\
& +(5 P|r| 4 S)^{2}\left[\left(7 S\left|r g_{P}\left(E_{4 S}-E_{5 P}+E_{7 S}\right) r\right| 7 S\right)\right. \\
& \left.+\left(7 S\left|r g_{P}\left(E_{4 S}+E_{5 P}-E_{7 S}\right) r\right| 7 S\right)\right] \\
& +(6 P|r| 4 S)^{2}\left[\left(7 S\left|r g_{P}\left(E_{4 S}-E_{6 P}+E_{7 S}\right) r\right| 7 S\right)\right. \\
& \left.+\left(7 S\left|r g_{P}\left(E_{4 S}+E_{6 P}-E_{7 S}\right) r\right| 7 S\right)\right] .
\end{aligned}
$$

Equations (2.11) and (2.12) are two alternative expressions for the same sum $S_{1}^{0}$. Thus they provide a good numerical test of our codes, which give results using these two equa-

TABLE II. Dispersion coefficients in a.u. for the ${ }^{39} \mathrm{~K}(4 s)-{ }^{39} \mathrm{~K}(5 d)$ asymptote.

\begin{tabular}{lccc}
\hline \hline & $\beta$ & $C_{5} \times 10^{2}$ & $C_{6} \times 10^{-5}$ \\
\hline$m=0$ & +1 & -86.150 & 4.0148 \\
& -1 & 86.150 & 4.0164 \\
$m= \pm 1$ & +1 & 57.433 & 3.5620 \\
& & & $3.3 \pm 0.3^{\mathrm{a}}$ \\
$m= \pm 2$ & -1 & -57.433 & 3.5609 \\
& +1 & -1.4358 & 2.1986 \\
& -1 & 1.4358 & 2.1992 \\
\hline \hline
\end{tabular}

${ }^{\mathrm{a}}$ Measurement of Wang et al. [1]. 
TABLE III. Dispersion coefficients in a.u. for the ${ }^{39} \mathrm{~K}(4 s)-{ }^{39} \mathrm{~K}(6 d)$ asymptote.

\begin{tabular}{lccc}
\hline \hline & $\beta$ & $C_{5} \times 10^{2}$ & $C_{6} \times 10^{-5}$ \\
\hline$m=0$ & +1 & -14.282 & 9.1057 \\
& -1 & 14.282 & 9.1068 \\
$m= \pm 1$ & +1 & 9.5212 & 8.0870 \\
& & & $10.1 \pm 0.7^{\mathrm{a}}$ \\
$m= \pm 2$ & -1 & -9.5212 & 8.0862 \\
& +1 & -2.3803 & 5.0275 \\
& -1 & 2.3803 & 5.0279 \\
\hline
\end{tabular}

${ }^{\mathrm{a}}$ Measurement of Wang et al. [1].

tions which agree to seven digits of accuracy. In a similar way, each of the sums may be reduced to an integral representation. One then must evaluate only atomic matrix elements containing a radial Green's function. This step may be done easily by using the Dalgarno-Lewis method [5] even in cases involving complex energies $[2,3]$.

\section{NUMERICAL RESULTS AND DISCUSSION}

The numerical results for the $C_{6}, C_{8}$, and $C_{10}$ coefficients corresponding to the ${ }^{39} \mathrm{~K}(4 s)-{ }^{39} \mathrm{~K}(7 s)$ asymptote are given in Table I, and those for the ${ }^{39} \mathrm{~K}(4 s)-{ }^{39} \mathrm{~K}(5 d, 6 d)$ asymptotes are given in Tables II and III. ${ }^{4}$ The theoretical computations have an uncertainty of $4 \%$ due mainly to the $2 \%$ experimental uncertainty of the static dipole polarizability of $\mathrm{K}$ [6], whose value our model potential has been constructed to reproduce, following the procedure of Ref. [3]. A discussion of the influence of the uncertainty of the measured static

\footnotetext{
${ }^{4}$ The coefficients $C_{k}$ have dimensions of energy $\times\left(\right.$ length ${ }^{k}$ and are given in the tables in a.u., where the energy unit in a.u. equals $27.211396 \mathrm{eV}$ and the length unit in a.u. equals $5.291772 \times 10^{-9}$ $\mathrm{cm}$.
}

dipole polarizability on the values of the calculated dispersion coefficients was given in Ref. [7] for the case of longrange interactions among three alkali-metal atoms. A similar discussion also holds for the dispersion coefficients of $\mathrm{K}$ dimers presented here. We notice that for the ${ }^{39} \mathrm{~K}(4 s)-{ }^{39} \mathrm{~K}(5 d, 6 d)$ asymptote, the $C_{5}$ coefficients have very small values in comparison with the $C_{6}$ coefficients. Thus the dominant contributions to the ${ }^{39} \mathrm{~K}(4 s)-{ }^{39} \mathrm{~K}(5 d, 6 d)$ asymptotes for $\mathrm{K}_{2}$ are given by the $1 / R^{6}$ term in Eq. (2.5) for $R<10^{7}$ a.u. Beyond $10^{7}$ a.u. the $C_{5}$ coefficients may begin to dominate. However, at such large distances the Casimir effect may become important and hence one expects a smooth transformation of the $1 / R^{5}$ term in Eq. (2.5) to one varying as the $1 / R^{6}$ power.

The experimental values [1] of the dispersion coefficients are obtained by analyzing the spectroscopic data of high vibrational levels. For the ${ }^{39} \mathrm{~K}(4 s)-{ }^{39} \mathrm{~K}(7 s)$ asymptote the measured vibrational spectrum has ${ }^{3} \Sigma_{g}^{+}$symmetry, while for the ${ }^{39} \mathrm{~K}(4 s)-{ }^{39} \mathrm{~K}(5 d, 6 d)$ asymptotes the vibrational spectrum has $1_{u}$ symmetry. The experimental values for the dispersion coefficients $C_{6}$ have been determined by using the single leading term potential model (cf. Ref. [8]). As shown in the tables, we find good agreement between theory and experiment for the leading terms of the ${ }^{39} \mathrm{~K}(4 s)-{ }^{39} \mathrm{~K}(7 s)$ and ${ }^{39} \mathrm{~K}(4 s)-{ }^{39} \mathrm{~K}(5 d)$ asymptotes. The disagreement between theory and experiment for the case of ${ }^{39} \mathrm{~K}(4 s)-{ }^{39} \mathrm{~K}(6 d)$ may be the result of neglect of finestructure contributions as well as the exchange interaction and Casimir effect in the long-range form of the potential curve used to extract the dispersion coefficients from the experimental data.

\section{ACKNOWLEDGMENT}

This work was supported by the U.S. Department of Energy, Office of Basic Energy Sciences, under Grant No. DEFG03-96ER14646.
[1] H. Wang, X. T. Wang, P. L. Gould, and W. C. Stwalley, Phys. Rev. Lett. 78, 4173 (1997).

[2] M. Marinescu and A. Dalgarno, Phys. Rev. A 52, 311 (1995).

[3] M. Marinescu, H. R. Sadeghpour, and A. Dalgarno, Phys. Rev. A 49, 982 (1994).

[4] C. Mavroyannis and M. J. Stephen, Mol. Phys. 5, 629 (1962); A. D. McLachlan, Proc. R. Soc. London, Ser. A 271, 387 (1963); see also the review by A. Dalgarno and W. D. Davi- son, Adv. At. Mol. Phys. 2, 1 (1966).

[5] A. Dalgarno and J. T. Lewis, Proc. R. Soc. London, Ser. A 233, 70 (1955).

[6] W. D. Hall and J. C. Zorn, Phys. Rev. A 10, 1141 (1974).

[7] M. Marinescu and A. F. Starace, Phys. Rev. A 55, 2067 (1997).

[8] R. J. LeRoy and R. B. Bernstein, J. Chem. Phys. 52, 3869 (1970). 